\title{
Valor predictivo del calculador de riesgo quirúrgico pediátrico National Surgical Quality Improvement Program - Pediatrics en pacientes de una unidad de neonatología: estudio retrospectivo correlacional
}

\author{
Predictive value of the National Surgical Quality Improvement Program - Pediatrics risk \\ calculator in neonatal patients. A retrospective correlational study
}

Manuel Gil-Vargas*, Dayana Pereira-Reyes y Ethel Lira-Marcial

Departamento de Cirugía Pediátrica, Hospital General de Puebla Dr. Eduardo Vázquez Navarro, Puebla, México

\begin{abstract}
Resumen
Objetivo: Comprobar el valor predictivo del calculador American College of Surgeons National Surgical Quality Improvement Program-Pediatric (NSQIP-P) como pronóstico de complicaciones en pacientes neonatos sometidos a cirugía. Método: Se realizó un estudio observacional, retrospectivo, transversal y correlacional. Se recopilaron los expedientes de los neonatos quirúrgicos de la unidad de neonatología del Hospital General de Puebla Dr. Eduardo Vázquez Navarro en el periodo comprendido entre enero de 2014 y mayo de 2017. Los datos se vaciaron en la plataforma arrojando porcentajes de estimación de riesgo. Se dio seguimiento a los pacientes por medio de los expedientes, observando su evolución para determinar qué complicaciones desarrollaron. Se correlacionaron los datos obtenidos en la plataforma con los hallados en el expediente y se determinó el valor predictivo de la escala. Resultados: De acuerdo con la clasificación de presencia o no de complicación, destacan por correlaciones sin significancia estadística. Presentan correlación positiva 78 casos (10.19\%), correlación negativa 279 casos (36.47\%) y correlación nula 408 casos (53.34\%). Conclusión: Se demostró que la escala tiene un bajo valor predictivo con tendencia a la sobrestimación; sin embargo, se corroboró su utilidad como parte del proceso de consentimiento informado en los neonatos quirúrgicos.
\end{abstract}

PALABRAS CLAVE: American College of Surgeons National Surgical Quality Improvement Program-Pediatric (NSQIP-P). Complicaciones posoperatorias. Neonatos.

\begin{abstract}
Objective: To verify the predictive value of the American College of Surgeons National Surgical Quality Improvement Program-Pediatric calculator as a prognosis for complications in neonatal patients undergoing surgery. Method: An observational, retrospective, cross-sectional and correlational study was carried out. The files of the surgical neonates of the neonatology unit of the General Hospital of Puebla Dr. Eduardo Vázquez Navarro in the period between January 2014 and May 2017. The data was emptied into the platform yielding percentages of risk estimation. The patients were followed up through the files observing their evolution to determine which complications developed. The data obtained in the platform was correlated with the data obtained in the file and the predictive value of the scale was determined. Results: According to the classification of presence or absence of complication, they stand out for correlations without statistical significance. Presenting positive correlation in 78 cases (10.19\%), negative correlation in 279 cases (36.47\%) and null correlation in 408 cases (53.34\%).
\end{abstract}

\author{
Correspondencia: \\ *Manuel Gil-Vargas \\ 5 sur, 5305-201 \\ C.P. 72440 , Puebla, Pue., México \\ E-mail: gilvm@yahoo.com
}

Fecha de recepción: 12-07-2018

Fecha de aceptación: 15-12-2018

DOI: $10.24875 / C I R U .18000593$
Cir Cir. 2019;87:308-312

Contents available at PubMed www.cirugiaycirujanos.com 
Conclusion: It was shown that the scale has a low predictive value with a tendency to overestimate, however, its usefulness was corroborated as part of the informed consent process in surgical neonates.

KEY WORDS: American College of Surgeons National Surgical Quality Improvement Program-Pediatric. Neonates. Postsurgical complications.

\section{Introducción}

En enero de 1994 se crea el programa de orientación a la calidad quirúrgica National Surgical Quality Improvement Program (NSQIP)', en los EE.UU., debido a un incremento en la tasa de morbilidad y mortalidad de los Veterans Administration Medical Centers, con el fin de disminuir el riesgo posquirúrgico. Posteriormente, al ser implementado en 132 hospitales se observó una reducción del $47 \%$ y del $43 \%$ en la mortalidad y la morbilidad, respectivamente, en los 30 días posteriores a la intervención quirúrgica².

EI NSQIP es un programa diseñado con el propósito de medir y mejorar la calidad quirúrgica de las instituciones mediante una plataforma que calcula el riesgo ajustado de complicaciones para cada paciente en los 30 días posteriores a la cirugía ${ }^{2}$.

Actualmente, en todo el mundo existe un gran enfoque en el desarrollo de herramientas de medición y mejora de la calidad en todas las áreas de la medicina, con el fin de mejorar el servicio y reducir las complicaciones, provocando el desarrollo de varias herramientas para la evaluación de la calidad, en especial en al ámbito quirúrgico ${ }^{3}$. Debido a la gran relevancia de estas herramientas, es importante que evaluemos su efectividad y precisión poniéndolas a prueba en diferentes poblaciones.

Existen pocos estudios aleatorizados en niños centrados en medir la calidad quirúrgica, en gran medida debido a ciertos factores propios de las cohortes, como son el reducido número de cirugías y la poca frecuencia con que se realizan ${ }^{3,4}$. Como se muestra en los resultados de Polites, et al. ${ }^{3}$, comparando entre el NSQIP-P y el Agency of Health Care Research and Quality - Pediatric Quality Indicators, la baja frecuencia de complicaciones posoperatorias pediátricas no es suficiente para medir de manera puntual la calidad en los procesos de cirugía pediátrica. Los valores predictivos de ambos métodos fueron similarmente bajos, del 7.9 y el $8.0 \%$, respectivamente, por lo que recomiendan el uso de otras medidas que coadyuven a la evaluación de la calidad. Por su parte, Bucher, et al. ${ }^{4}$ analizan la morbilidad y la mortalidad utilizando la base de datos disponible de la plataforma NSQIP, y encuentran en pacientes neonatos una morbilidad del $44.4 \%$ y una mortalidad del $6.9 \%$, valores significativamente mayores sin discriminación entre pretérmino y a término frente a los no neonatos con valores de morbilidad del $6.4 \%$ y de mortalidad del $0.1 \%$, por lo que concluyen que debe existir diferenciación para el cálculo de los riesgos entre los neonatos y la población pediátrica en general. Langham, et al. ${ }^{5}$ realizan el análisis de las variables que influyen mayormente en la mortalidad, utilizando su base de datos en NSQIP-P del Le Bonheur Children Hospital. Comparándola con base de datos propia del NSQIP-P, los resultados sobre las variables muestran que, de las 21 que incluye el NSQIP, siete tienen valor predictivo de mortalidad: estatus neonatal, soporte respiratorio, soporte inotrópico, lesiones cerebrovasculares, previa intervención cardiaca y unidad de trabajo relativo.

Existe actualmente una tendencia creciente a involucrar a los familiares en el proceso médico-quirúrgico ${ }^{5}$. La plataforma National Surgical Quality Improvement Program - Pediatric (NSQIP-P) $)^{6}$ ofrece un panorama más detallado sobre los posibles resultados de la cirugía y de esta manara tranquiliza a los padres, obteniendo una comunicación eficaz y empática con el médico. Las características de esta base de datos permiten no solo ofrecer a los familiares información clara y transparente sobre los procedimientos que se llevan a cabo en la sala de quirófano, sino también recabar información importante para obtener datos estadísticos confiables que pueden mejorar la calidad en los centros hospitalarios ${ }^{5}$. De igual forma, puede contribuir a tomar decisiones considerando el riesgo y el beneficio, determinando si es viable operar a un paciente o no. Kraemer, et al. ${ }^{6}$ analizan la base de datos NSQIP-P para conocer la incidencia de eventos posquirúrgicos, proporcionando así una valiosa herramienta predictiva.

La calculadora de riesgo quirúrgico del American College of Surgeons estima la probabilidad de un resultado desfavorable (como una complicación o muerte) después de la cirugía. El riesgo se estima basándose en la información que el paciente da al proveedor de atención médica acerca de su historial de salud. Las estimaciones se calculan utilizando datos de un gran 
número de pacientes que tuvieron un procedimiento quirúrgico similar al que el paciente puede tener?.

Calder, et al. ${ }^{8}$ evaluaron la relación entre los resultados de NSQIP-P y las Traditional Morbidity and Mortality Conferences, y concluyeron que existe poca correlación entre los resultados de ambos métodos para capturar complicaciones, especialmente complicaciones menos graves, siendo mayor la correlación entre las complicaciones más graves. Ellos atribuyen estas discordancias a las fallas en la detección de dichas complicaciones por parte del personal médico en entrenamiento, es decir, los residentes. EI NSQIP-P ha probado dar resultados en población fuera de los EE.UU., en países como Canadá, proporcionando datos de alta calidad que pueden utilizarse para mejorar el desempeño de los centros hospitalarios ${ }^{9}$. Estos procesos que propone el NSQIP-P requieren un trabajo en equipo multidisciplinario, participación sistemática de los interesados, métodos de investigación clínica y mejora del proceso a través del compromiso y el cambio cultural ${ }^{10}$. Actualmente existen pocos estudios que evalúen la confiabilidad del uso predictivo del calculador de riesgo del NSQIP.

\section{Método}

Se realizó un estudio observacional, retrospectivo, trasversal y correlacional en las instalaciones del Hospital General de Puebla Dr. Eduardo Vázquez Navarro, de los Servicios de Salud del Estado de Puebla. El marco muestral se conformó por 85 procedimientos obtenidos de 127 expedientes revisados del archivo de dicho hospital que cumplieron con los criterios de selección. Como criterios de inclusión se seleccionaron expedientes de pacientes de edad neonatal del archivo de enero de 2014 a junio de 2017 sometidos a procedimientos quirúrgicos en la unidad de estudio. Como criterios de exclusión se consideraron los expedientes incompletos, y como criterios de eliminación, los expedientes que contaran con procedimientos quirúrgicos no citados en el calculador NSQIP-P.

Se recopilaron del archivo los expedientes del periodo de enero de 2014 a junio de 2017 de pacientes neonatos, considerando como tales los infantes nacidos a término menores de 29 días de vida o menores de 37 semanas de gestación al momento del nacimiento y con menos de 50 semanas de vida extrauterina, de acuerdo con los criterios de la plataforma NSQIP-P.

Se obtuvieron de los expedientes los datos requeridos para el cálculo del riesgo con la plataforma NSQIP-P en su hoja de recolección de datos. Se realizó el vaciado de los datos a la plataforma en
Tabla 1. Tabla de Coincidencias.

\begin{tabular}{llll}
\hline Clasificación & Código & NSQIP-P & Expediente \\
\hline Coincidencia 1 & C1 & Alto & Presente \\
Coincidencia 2 & C2 & Bajo & Ausente \\
Neutro/No & C0 & Medio & Ausente \\
Sobreestima & C0+ & Alto & Ausente \\
Subestima $>$ & $\mathrm{CO}$ & Bajo & Presente \\
Subestima - & $\mathrm{CO}$ & Medio & Presente \\
No & $\mathrm{COz}$ & Suma de las no coincidencias \\
coincidencia & & & \\
\hline
\end{tabular}

línea, la cual arrojó de manera automática los porcentajes de estimación del riesgo quirúrgico. Estos datos se transcribieron a la base de datos realizada en el programa de análisis estadístico SPSS.

Para el análisis de correlación entre las variables se nombró coincidencia 1 (C1) a las complicaciones presentes y al valor del calculador NSQIP-P de alto riesgo; coincidencia 2 (C2) a la no presencia de riesgo y al valor de bajo riesgo según la plataforma; y coincidencia 0 (C0) cuando no se presentó la complicación y el riesgo fue medio. Cuando la complicación no se presentó y el calculado sobreestimó como alto riesgo se denominó $\mathrm{C} 0+$; cuando la complicación se presentó y el calculador subestimó como bajo riesgo, $\mathrm{C} 0<$; y cuando la complicación se presentó y el calculador calificó como bajo riesgo, C0-. Para fines de conocer cuándo el calculador sobreestima, subestima o no coincide, se concentran todas las no coincidencias como COz. Esta clasificación se muestra en la tabla 1.

\section{Resultados}

En el estudio se analizaron 85 procedimientos quirúrgicos correspondientes a 148 expedientes, lo cuales cumplieron con los criterios de selección; se excluyeron 43 y se eliminaron 12. La población estudiada fue de 53 hombres (62.4\%) y 32 mujeres (37.6\%). La prevalencia en el tipo de cirugía fue similar, siendo la cirugía emergente la más realizada (45 pacientes; $52.9 \%$ ). En cuanto a la transferencia, hubo una marcada prevalencia en pacientes transferidos de la unidad de cuidados intensivos (69; 70.0\%). La clasificación American Society of Anesthesiologists (ASA) más presentada fue la tipo III, con un valor de $38(44.7 \%)$, seguida de la tipo II con un valor de 33 (38.8\%); destaca la presencia de un único caso (1.2\%) de tipo IV. La clasificación de las heridas que se encontró en la mayor parte de los 
Tabla 2. Resultado de concentrados.

\begin{tabular}{|c|c|c|c|c|c|c|c|c|c|c|}
\hline Código & $\mathrm{CC}$ & NEU & CCAR & ISQ & ITU & TV & FR & RIT & MRT & Total \\
\hline $\mathrm{C} 1$ & 28 & 5 & 19 & 0 & 7 & 0 & 7 & 5 & 7 & 78 \\
\hline C2 & 18 & 36 & 24 & 31 & 26 & 48 & 37 & 33 & 26 & 279 \\
\hline CO & 12 & 7 & 5 & 24 & 14 & 5 & 9 & 6 & 9 & 91 \\
\hline $\mathrm{CO}+$ & 2 & 29 & 16 & 19 & 31 & 32 & 25 & 37 & 38 & 229 \\
\hline $\mathrm{CO}<$ & 16 & 6 & 19 & 6 & 6 & 0 & 7 & 4 & 5 & 69 \\
\hline $\mathrm{CO}-$ & 9 & 2 & 2 & 5 & 1 & 0 & 0 & 0 & 0 & 19 \\
\hline$R=$ & 0.42 & -0.04 & 0.15 & 0.00 & 0.09 & -0.09 & 0.16 & 0.06 & 0.10 & 765 \\
\hline
\end{tabular}

procedimientos fue de tipo limpia $(52 ; 61.2 \%)$. La presencia de sepsis 48 horas antes de la cirugía se encontró en 17 casos, subdivididos en síndrome de respuesta inflamatoria sistémica $(9 ; 10.6 \%)$, sepsis $(7$; $8.2 \%)$ y shock $(1 ; 1.2 \%)$, siendo infrecuente su presencia en $68(80.0 \%)$. La necesidad de ventilación mecánica fue de 23 (27.1\%). Los trastornos neuromusculares se objetivaron en $16(18.8 \%)$, y los hematológicos, en $9(10.6 \%)$. El uso de oxígeno suplementario fue la única variable en la que destacó la presencia sobre la ausencia, con 49 (57.6\%) casos.

Los resultados de acuerdo con la clasificación de presencia o no de complicación destacan por presentar correlaciones sin significancia estadística. La correlación fue nula para infección del sitio quirúrgico $(r=0.00)$, y muy baja para las variables neumonía $(r=-0.04)$, complicaciones cardiacas $(r=0.15)$, infección del tracto urinario $(r=0.09)$, trombosis venosa $(r=-0.09)$, falla renal $(r=0.16)$, reintubación $(r=0.06)$ y muerte $(r=0.10)$. La correlación entre la variable cualquier complicación y los datos del calculador NSQIP-P es la única positiva, con un valor de correlación muy bajo. $\mathrm{La} \mathrm{C} 1$ con la variable $\mathrm{CC}$ se presentó en 28 ocasiones, siendo la mayor coincidencia con el $32.94 \%$ de los resultados; C2 con CC se presentó en 18 de los casos (21.18\%); y COz (suma de las no coincidencias) tuvo el mayor número de resultados: 39 (45.88\%). En la complicación de neumonía, la C1 fue una de las más bajas, con solo cinco resultados positivos (5.88\%), en contraste con la $\mathrm{C} 2$, en la que la coincidencia entre los resultados y el calculador fue de $36(42.35 \%)$; sin embargo, al igual que con la variable CC, la coincidencia $\mathrm{COz}$ fue la de mayor numero, con 44 (51.76\%), sin coincidir con el calculador. En la complicación de infección del sitio quirúrgico, la coincidencia $\mathrm{C} 1$ fue nula, con $0(0.00 \%)$, y la $\mathrm{COz}$ fue de 54 (63.53\%), coincidiendo estos resultados con la variable de infección del tracto urinario, con una $\mathrm{COz}$ de 52 (63\%).

En la trombosis venosa, $\mathrm{C} 1$ es $0(0.00 \%)$. En falla renal, los valores de $\mathrm{C} 2$ y $\mathrm{COz}$ son similares, con $37(43.53 \%)$ y 41 (48.52\%). Para los procedimientos que necesitaron reintubación, la COz fue de 47 (55.29\%). Finalmente, para la mortalidad C1 obtuvo 7 (8.24\%), C2 obtuvo 26 (30.59\%) y C0z obtuvo 52 (61.18\%); valores que explican el porqué de las correlaciones bajas o nulas, ya que los valores de C1 para todas las variables es solo del $10.19 \%$, mientras que los de C2 son del $36.47 \%$ y los de $\mathrm{COz}$ son del $53.33 \%$. Los resultados concentrados se muestran en la tabla 2.

\section{Discusión}

Ante el gran interés por el desarrollo de herramientas de mejora de la calidad, que tienen como objetivo reducir las tasas de complicaciones, la plataforma NSQIP-P ha sido una de las más utilizadas con buenos resultados para valorar la morbilidad y la mortalidad, como se mostró en los en trabajos de Bucher, et al. ${ }^{4}$. También Langham, et al. ${ }^{5}$ destacansu utilidad en las variables directamente asociadas a la mortalidad. El NSQIP-P destaca como herramienta que ofrece un panorama detallado de los posibles resultados que se muestran a los padres como parte de un consentimiento informado transparente. Coinciden con esta utilidad Kraemer, et al. ${ }^{6}$, quienes concluyen que calcular el riesgo es una herramienta fácil de usar que ofrece información adicional para mejorar el proceso de toma de decisión, pero valoran al calculador NSQIP con poca certeza de estimación, con una pequeña tendencia a subestimar, a diferencia de nuestros resultados, en los que obtenemos una subestimación $\mathrm{CO}<$ del $9.01 \%$ y una subestimación C0- del $2.48 \%$, con tendencia a la sobreestimación C0+ en el $29.93 \%$ de los casos, sin 
dejar de lado que nuestros resultados van hacia una tendencia superior del conjunto $\mathrm{COz}$. Coincidiendo, Polites, et al. ${ }^{3}$ muestran unos resultados bajos del NSQIP-P como valor predictivo por ser infrecuentes los eventos adversos en las cohortes pediátricas, por lo que debería cuestionarse el uso de estos valores para evaluar la calidad quirúrgica. Reforzando esta visión, Zhang, et al. ${ }^{11}$ comparan el NSQIP-P con las Traditional Morbidity and Mortality Conferences de Canadá, y concluyen que las complicaciones que se presentan en el posquirúrgico no son necesariamente atribuibles al procedimiento quirúrgico, por lo cual sugieren que se extienda el tiempo de valoración en que se presentan las complicaciones hasta 180 o 365 días, así como la inclusión de otras especialidades, como cardiología y traumatología.

De acuerdo con la Norma Oficial Mexicana del expediente clínico $^{12}$, se define el consentimiento informado como «los documentos escritos, signados por el paciente o su representante legal o familiar más cercano en vínculo, mediante los cuales se acepta un procedimiento médico o quirúrgico con fines diagnósticos, terapéuticos, rehabilitatorios, paliativos o de investigación, una vez que se ha recibido información de los riesgos y beneficios esperados para el paciente». El consentimiento informado, más que un documento, es un acto mediante el cual se proporciona una información detallada y completa a un sujeto sobre los pormenores de los procedimientos, enfatizando los riesgos a los que se expone. Ese mismo acto en la atención médica se basa en la información descriptiva y detallada que se hace al usuario sobre su enfermedad, los procedimientos diagnósticos y de tratamiento, las alternativas, las complicaciones, y las secuelas o los riesgos que conllevan dichos procedimientos, para que de una manera consciente, informada, responsable y con plena libertad decida someterse a ello, aceptando los riesgos que implique ${ }^{13}$. Por esto, es crucial la integración de herramientas como el NSQIP-P, que han probado ser de gran utilidad para el cumplimiento de esta ley y una buena relación entre médico y paciente.

\section{Conclusión}

De todo lo anterior podemos concluir que el calculador de riesgo del NSQIP-P es una escala diseñada con el propósito de servir en el proceso de toma de decisión y consentimiento informado, para lo cual sirve en gran medida debido a su tendencia a la sobreestimación; sin embargo, no debe utilizarse como herramienta predictiva, sino como herramienta preventiva.

\section{Financiamiento}

Sin financiamiento.

\section{Conflicto de intereses}

Los autores declaran que no existe conflicto de intereses.

\section{Responsabilidades éticas}

Protección de personas y animales. Los autores declaran que para esta investigación no se han realizado experimentos en seres humanos ni en animales.

Confidencialidad de los datos. Los autores declaran que han seguido los protocolos de su centro de trabajo sobre la publicación de datos de pacientes.

Derecho a la privacidad y consentimiento informado. Los autores declaran que en este artículo no aparecen datos de pacientes.

\section{Bibliografía}

1. Khuri SF, Daley J, Henderson W, Hur K, Demakis J, Aust JB, et al. The Department of Veterans Affairs' NSQIP: the first national, validated, outcome-based, risk-adjusted, and peer-controlled program for the measurement and enhancement of the quality of surgical care. National VA Surgical Quality Improvement Program. Ann Surg. 1998;228:491-507.

2. Dillon P, Hammermeister K, Morrato E, Kempe A, Oldham K, Moss L, et al. Developing a NSQIP module to measure outcomes in children's surgical care: opportunity and challenge. Semin Pediatr Surg. 2008;17:131-40.

3. Polites SF, Habermann EB, Zarroug AE, Wagie AE, Cima RR, Wiskerchen $\mathrm{R}$, et al. A comparison of two quality measurement tools in pediatric surgery - The American College of Surgeons National Surgical Quality Improvement Program-Pediatric versus the Agency for Healthcare Research and Quality Pediatric Quality Indicators. J Pediatr Surg. 2015;50:586-90.

4. Bucher BT, Duggan EM, Grubb PH, France DJ, Lally KP, Blakely ML. Does the American College of Surgeons National Surgical Quality Improvement Program Pediatric provide actionable quality improvement data for surgical neonates? J Pediatr Surg. 2016;51:1440-4.

5. Langham MR, Walter A, Boswell TC, Beck R, Jones TL. Identifying children at risk of death within 30 days of surgery at an NSQIP pediatric hospital. Surgery. 2015;158:1481-91.

6. Kraemer K, Cohen ME, Liu Y, Barnhart DC, Rangel SJ, Saito JM, et al. Development and evaluation of the American College of Surgeons NSQIP Pediatric surgical risk calculator. J Am Coll Surg. 2016:223:685-93.

7. ACS Pediatric Risk Calculator - Home Page. (Consultado el 5 de junio de 2018.) Disponible en: http://riskcalculator.facs.org/peds/Patientlnfo.jsp .

8. Calder BW, Sakran JV, Streck CJ, Cina RA. What happened to the complication? The importance of ACS NSQIP Pediatric in optimizing quality improvement initiatives for resident education. J Surg Educ. 2017; 74:431-6. Disponible en: http://dof.gob.mx/nota_detalle_popup.php?codigo $=5272787$.

9. Skarsgard ED, Bedford J, Chan T, Whyte S, Afshar K. ACS National Surgical Quality Improvement Program: targeting quality improvement in Canadian pediatric surgery. J Pediatr Surg. 2014;49:682-7.

10. Piatt JH. Thirty-day outcomes of cerebrospinal fluid shunt surgery: data from the National Surgical Quality Improvement Program-Pediatrics: clinical article. J Neurosurg Pediatr. 2014;14:179-83.

11. Zhang JX, Song D, Bedford J, Bucevska M, Courtemanche DJ, Arneja JS. What is the best way to measure surgical quality? Comparing the American College of Surgeons National Surgical Quality Improvement Program versus Traditional Morbidity and Mortality Conferences. Plast Reconstr Surg. 2016;137:1242-50.

12. Norma Oficial Mexicana NOM-004-SSA3-2012, del expediente clínico. 2012;(15):23.

13. López IFD. Aspectos legales y éticos del consentimiento informado en la atención médica en México. Rev Mex Patol Clin. 2001;48:4. 